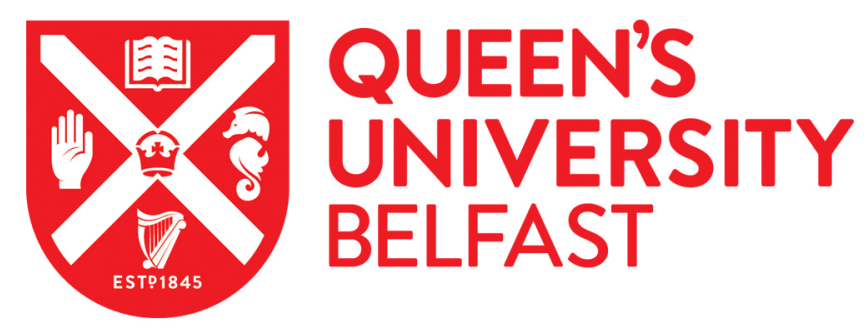

\title{
Structural damage diagnosis of steel truss bridges by outlier detection
}

Kim, C. W., Kitauchi, S., Chang, K. C., Mcgetrick, P. J., Sugiura, K., \& Kawatani, M. (2014). Structural damage diagnosis of steel truss bridges by outlier detection. In G. Deodatis, B. R. Ellingwood, \& D. M. Frangopol (Eds.), Safety, Reliability, Risk and Life-Cycle Performance of Structures and Infrastructures - Proceedings of the 11th International Conference on Structural Safety and Reliability, ICOSSAR 2013 (pp. 4631-4638). CRC Press. https://www.crcpress.com/Safety-Reliability-Risk-and-Life-Cycle-Performance-of-Structures-and/DeodatisEllingwood-Frangopol/9781138000865

Published in:

Safety, Reliability, Risk and Life-Cycle Performance of Structures and Infrastructures - Proceedings of the 11th International Conference on Structural Safety and Reliability, ICOSSAR 2013

\section{Document Version:}

Peer reviewed version

Queen's University Belfast - Research Portal:

Link to publication record in Queen's University Belfast Research Portal

\section{Publisher rights}

(c) 2014 CRC Press/Balkema.

The final version was published in Safety, Reliability, Risk and Life-Cycle Performance of Structures and Infrastructures

\section{General rights}

Copyright for the publications made accessible via the Queen's University Belfast Research Portal is retained by the author(s) and / or other copyright owners and it is a condition of accessing these publications that users recognise and abide by the legal requirements associated with these rights.

Take down policy

The Research Portal is Queen's institutional repository that provides access to Queen's research output. Every effort has been made to ensure that content in the Research Portal does not infringe any person's rights, or applicable UK laws. If you discover content in the Research Portal that you believe breaches copyright or violates any law, please contact openaccess@qub.ac.uk. 


\title{
Structural damage diagnosis of steel truss bridges by outlier detection
}

\author{
C.W. Kim, S. Kitauchi, K.C. Chang, P.J. McGetrick \& K. Sugiura \\ Dept. of Civil and Earth Resources Eng., Graduate School of Eng., Kyoto University, Kyoto 615-8540, Japan \\ M. Kawatani \\ Dept. of Civil Eng., Graduate School of Eng., Kobe University, Kobe 657-8501, Japan
}

\begin{abstract}
This study discusses structural damage diagnosis of real steel truss bridges by measuring traffic-induced vibration of bridges and utilizing a damage indicator derived from linear system parameters of a time series model. On-site damage experiments were carried out on real steel truss bridges. Artificial damage was applied to the bridge by severing a truss member with a cutting machine. Vehicle-induced vibrations of the bridges before and after applying damage were measured and used in structural damage diagnosis of the bridges. Changes in the damage indicator are detected by Mahalanobis-Taguchi system (MTS) which is one of multivariate outlier analyses. The damage indicator and outlier detection was successfully applied to detect anomalies in the steel truss bridges utilizing vehicle-induced vibrations. Observations through this study demonstrate feasibility of the proposed approach for real world applications.
\end{abstract}

\section{INTRODUCTION}

The collapse of the I-35W Mississippi River bridge in Minneapolis, Minnesota, USA on August 1, 2007, was an unprecedented shock to the civil engineering community (National Transportation Safety Board 2008). After the event, even in Japan, bridge inspections have observed damage at members of steel truss bridges as shown in Figure 1 (Yamada 2008, Japan Road Association 2009). The importance and need for bridge inspection and monitoring has increasingly become more apparent in the aftermath of these events. In the field of inspection and monitoring, bridge owners have been drawn to vibrationbased structural health monitoring (SHM) since vibration-based SHM is expected to provide a more efficient way of SHM compared to visual inspections.

Most precedent studies on bridge health monitoring (BHM) specifically examine the change of modal properties and quantities of bridges (Salawu 1997, Doebling et al. 1998, Deraemaeker et al. 2007). The fundamental concept of this technology is that modal parameters are functions of structure's physical properties and a change in these physical properties, such as reduced stiffness resulting from damage, will detectably change these modal properties. In identifying modal parameters, many studies also utilize a linear time-series model (Shinozuka et al. 1982, He \& De Roeck 1997, Carden \& Brownjohn 2008, Kim et al. 2012). Existing studies show the

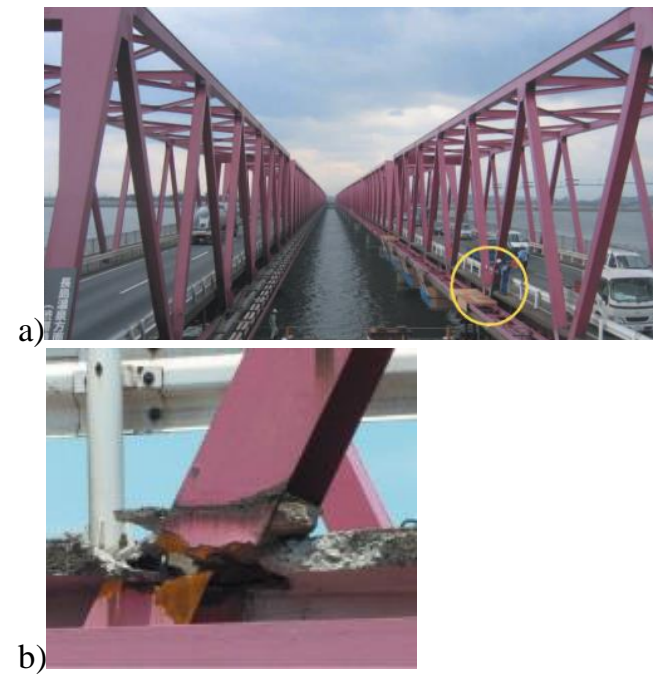

Figure 1. Rupture observed in a truss member due to corrosion: a) general view; b) rupture in a diagonal member.

feasibility of detecting bridge damage from changes in dynamic parameters, focusing on changes of modal frequencies, damping and mode shapes under a controlled condition (e.g. Catbas \& Aktan 2002, Lee \& Yun 2006).

For truss bridges, most of the existing studies investigate damage detection by means of numerical simulations and laboratory experiments (e.g. Kopsaftopoulos \& Fassois 2010, Wang et al. 2012). Yoshioka et al. (2011) investigated how damage in a real truss bridge member affects the frequency change of the bridge through a hammering test, and concluded that decreases in higher modes over 
$100 \mathrm{~Hz}$ were observed due to damage, although a lot of effort is required to perform the hammering test.

However, there exists a drawback of modal parameter-based bridge diagnosis using time series models: the optimal time series model for vibration responses of bridge structures usually comprises a higher order term and as a result, the optimal model detects even numerical parameters which cause spurious system frequencies and damping constants. In fact, those spurious system parameters make it difficult to choose proper modal parameters affected by structural damage. Nair et al. (2006) investigated feasibility of a damage-sensitivity feature derived from linear system parameters utilizing data from the ASCE benchmark test on a scaled building. Kim et al. (2013) also examined the feasibility of the damage-sensitivity feature derived from linear system parameters but the study considers damage detection of a scaled bridge under a passing model vehicle. Both investigations show successful application of the damage-sensitivity feature for damage detection within the laboratory scale.

This study investigates the feasibility of vibration-based damage detection of real steel truss bridges utilizing traffic-induced vibration of the bridges. On-site moving vehicle experiments on a real nine-span-continuous truss bridge and a singlespan truss bridge were carried out. Both bridges are steel bridges and are planned to be removed. It is noteworthy that the on-site experiment on the continuous-span bridge focuses on the detection of damage in a diagonal member under heavy vehicleinduced vibration. On the other hand, for the singlespan bridge, feasibility of fault detection of the bridge with step-by-step damage applied to vertical members is investigated, with vibrations induced by a light moving vehicle.

This study considers a damage indicator derived from linear system parameters of a time series model identified from traffic-induced vibration data as a damage-sensitive feature. The Mahalanobis-Taguchi system (MTS) (Taguchi \& Jugulum 2000) which is a multivariate statistical pattern recognition method (e.g. Sohn et al. 2001) is used to cope with any variation of identified results caused by uncertainties in the health monitoring and to emphasize potential change in the identified damage indicator due to damage. The outliers crossing the threshold are counted and this information is used to make a decision on bridge condition.

\section{ON-SITE MOVING VEHICLE EXPERIMENTS ON STEEL TRUSS BRIDGES}

On-site damage experiments were conducted on real steel truss bridges shown in Figures 2 and 3: a ninespan-continuous steel-truss bridge (hereafter, Bridge-A) is shown in Figure 2; and a single-span steel-truss bridge (hereafter, Bridge-B) is shown in Figure 3. Only the sixth span from the A1 abutment of Bridge-A was examined. The roadway roughness condition was poor since neither bridge has been maintained for quite a while.

\subsection{On-site experiment on continuous-span steel truss bridge}

For Bridge A, two different damage scenarios, healthy and damage by severing the diagonal member, were considered. 14 accelerometers were installed to measure vertical acceleration responses and accelerometers were relatively more densely deployed near the damage member as shown in Figure 4. Four different sensor groups were considered in the damage detection, which appear in Figure 4 as Group 1, Group 2, Group 3 and Group 4. It is noteworthy that the design tensile force of the damage member under dead load is $635 \mathrm{kN}$.

A cargo truck of model LKG-CD5ZA produced by UD Trucks Corp was used in the experimental campaign over two days, although vehicle weights were slightly altered from $253 \mathrm{kN}$ to $258 \mathrm{kN}$. However, effects of the small difference in the vehicle's axle load to the bridge response were negligible. Photoelectric switches mounted on the entrance, center and exit of the span were used to estimate location and average speed of the passing vehicle.

A vehicle-drop test showed that natural frequencies of the vehicle were $3.0 \mathrm{~Hz}$ and $3.5 \mathrm{~Hz}$ for bounce motions of the front axle and rear axle respectively. Those for hop motions appeared around $12 \mathrm{~Hz}$ for the front axle and $14 \mathrm{~Hz}$ for the rear axle. Vehicle-induced vibrations of the bridges before and after severing the member were measured and utilized in fault detection of the bridge.

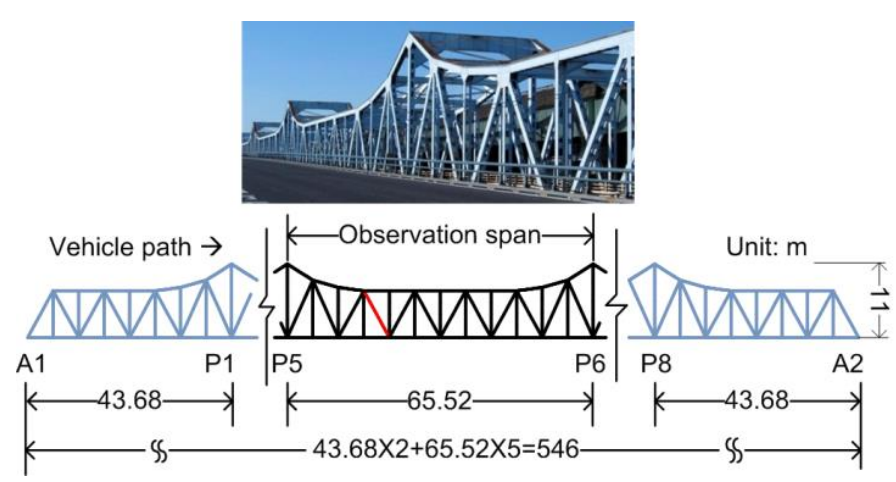

Figure 2. Elevation views of observation bridge, Bridge-A.

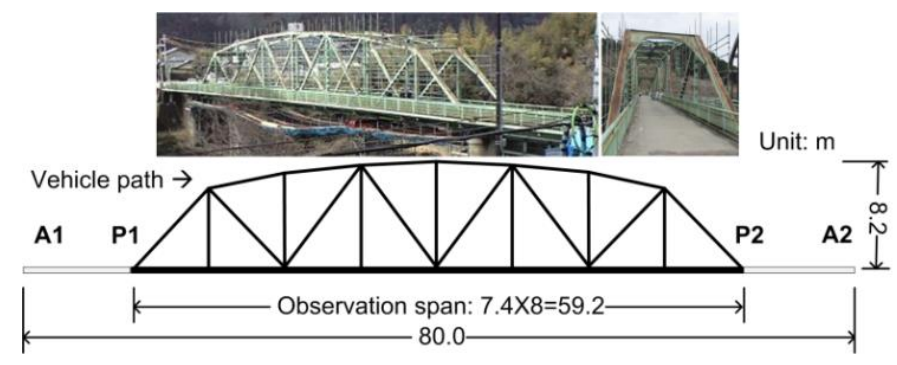

Figure 3. Elevation views of observation bridge, Bridge-B. 


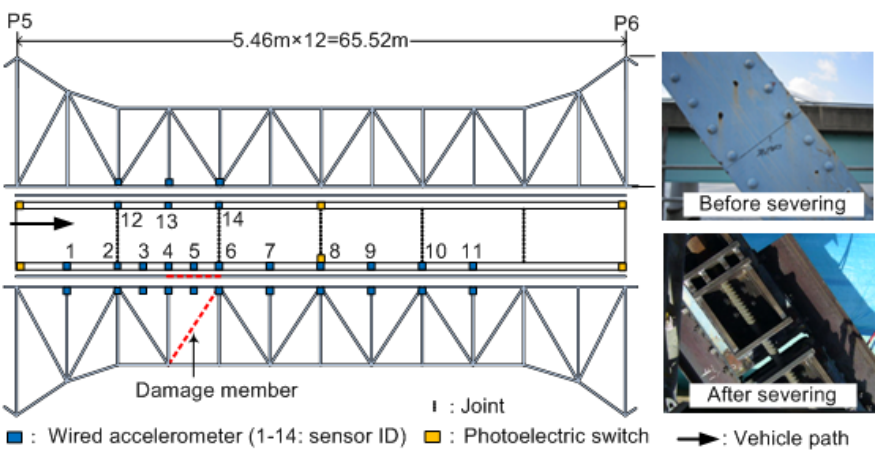

Group 1: Sensor group comprising sensors from 4 and 6 to 11 is considered in the analysis. Group 2: Sensor group comprising sensors from 4, 7, 9 and 10 is considered in the analysis. Group 3: Sensor group comprising two adjacent sensors such as $A(1,2), B(2,3), C(3,4)$, $\mathrm{D}(4,5), \mathrm{E}(5,6), \mathrm{F}(6,7), \mathrm{G}(7,8), \mathrm{H}(8,9), \mathrm{I}(9,10), \mathrm{J}(10,11), \mathrm{K}(12,13)$ and $\mathrm{L}(13,14)$ is $\mathrm{D}(4,5), E(5,6), F(6,7), G(7,8), H(8,9), 1(9,10), J(10,11), K(12,13)$ and $L(13,14)$ is

Group 4: Sensor group comprising two adjacent sensors such as $\mathrm{A}(1,2), \mathrm{BC}(2,4), \mathrm{DE}(4,6)$, $F(6,7), G(7,8), H(8,9), I(9,10), J(10,11), K(12,13)$ and $L(13,14)$ is considered in the analysis (deploying sensors only at nodal points).

Figure 4. Sensor deploying map and artificial damage of Bridge-A.

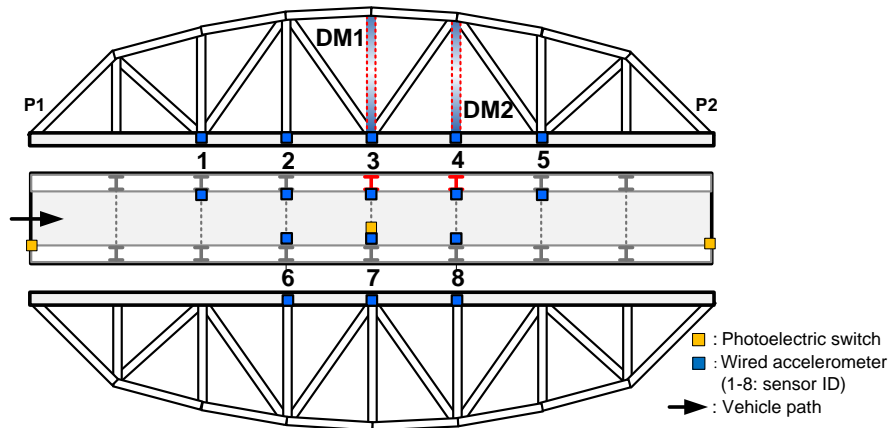

Group 1: Sensor group comprising all the sensors is considered in the analysis. Group 2: Sensor group comprising sensors from 1 to 5 is considered in the analysis. Group 3: Sensor group comprising sensors from 6 to 8 is considered in the analysis. Group 4: Sensor group comprising two adjacent sensors such as $A(1,2), B(2,3), C(3,4)$ $D(4,5), E(6,7)$ and $F(7,8)$ is considered in the analysis.

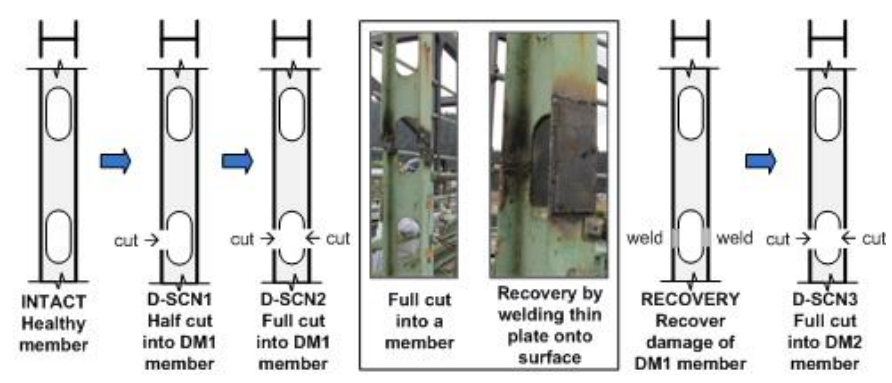

Figure 5. Sensor deploying map and artificial damage of Bridge-B.

During the experiment, 18 runs were carried out for the intact bridge which comprises 4 runs under vehicle speed of $10 \mathrm{~km} / \mathrm{h}, 7$ runs under vehicle speed of $20 \mathrm{~km} / \mathrm{h}$ and 7 runs under vehicle speed of $40 \mathrm{~km} / \mathrm{h}$. For the damage bridge, 15 runs were carried out, which comprises 3 runs under vehicle speed of $10 \mathrm{~km} / \mathrm{h}, 6$ runs under vehicle speed of $20 \mathrm{~km} / \mathrm{h}$ and 6 runs under vehicle speed of $40 \mathrm{~km} / \mathrm{h}$.

\subsection{On-site experiment on single-span steel truss bridge}

For Bridge B, five different damage scenarios were considered: Intact (hereafter INTACT); cut half of the section of DM1 member (Damage scenario 1, hereafter D-SCN1); cut the whole section of DM1 member (Damage scenario 2, hereafter D-SCN2); recover damage of DM1 member by welding thins plates onto the surface of the member (hereafter RECOVERY); and cut the whole section of DM2 member (Damage scenario 3, hereafter D-SCN3) as shown in Figure 5. 8 accelerometers were installed to measure vertical acceleration responses. Two accelerometers for the lateral responses were also installed at points 3 and 4 . However this study focused on the vertical responses only hence discussion on the lateral responses is omitted in this paper.

It is also noteworthy that the design tensile force of the DM1 member under dead load is around $34 \mathrm{kN}$, and that of the DM2 member is around $102 \mathrm{kN}$ due to dead load.

A van-type vehicle, Serena produced by Nissan, was used in the moving vehicle experiment. The weight of the vehicle is around $21 \mathrm{kN}$. In the experiment, 26 runs were carried out for the intact bridge which comprises 11 runs under vehicle speed of $30 \mathrm{~km} / \mathrm{h}, 10$ runs under vehicle speed of $40 \mathrm{~km} / \mathrm{h}$ and 5 runs under vehicle speed of $50 \mathrm{~km} / \mathrm{h}$. For the damage bridge, 12 runs for the D-SCN1 and 10 runs respectively for D-SCN2 and D-SCN3 under vehicle speed of $40 \mathrm{~km} / \mathrm{h}$ were performed for each damage scenario.

\section{OUTLIER ANALYSIS}

The Mahalanobis-Taguchi System (MTS) is adopted as a tool for multivariate outlier analysis, which is used in diagnostic applications to make quantitative decisions by constructing a multivariate measurement scale called Mahalanobis distance (Taguchi \& Jugulum 2000) (hereafter MD). In the MTS, the Mahalanobis space (MS) is obtained using the standardized variables of normal data. The MS can be used to discriminate normal and abnormal data.

In applying MTS, firstly we have to construct a measurement scale with MS as a reference: this is done using the data from a normal group and calculating their MDs, whose value should be close to 1 . The standardized normal data are obtained using Equation 1.

$$
z_{i}^{p}=\frac{x_{i}^{p}-\mu_{i}}{\sigma_{i}}
$$

where $z_{i}^{p}$ indicates the standardized $p$-th tuple of normal data for $i$-th variable; $x_{i}^{p}$, the $p$-th tuple of normal data for $i$-th variable; $\mu_{i}\left(=\frac{1}{n} \sum_{p=1}^{n} x_{i}^{p}\right)$, mean value of the $i$-th variable defined as; and $\sigma_{i}\left(=\frac{1}{n} \sqrt{\sum_{p=1}^{n}\left(x_{i}^{p}-\mu_{i}\right)^{2}}\right)$, the standard deviation of $i$-th variable. If $\mathbf{z}_{p}=\left(z_{1}^{p}, z_{2}^{p}, \cdots, z_{k}^{p}\right) \quad$ and 
$\mathbf{C} \in \mathbb{R}^{k \times k}$ denotes correlation matrix for $k$ standardized variables, then MD calculated for the $p$-th tuple of normal data in a sample size $n$ with $k$ variable is given by

$$
M D_{p}=D_{p}^{2}=\frac{1}{k} \mathbf{Z}_{p}^{T} \mathbf{C}^{-1} \mathbf{Z}_{p}
$$

Next, the signal space is obtained from abnormal data or newly monitored data. Abnormal data are also standardized utilizing mean and standard deviation values of the normal data as

$y_{i}^{p}=\frac{w_{i}^{p}-\mu_{i}}{\sigma_{i}}$

The $\overline{M D}_{p}$ of the normalized abnormal data in the signal space can be defined by Equation 4 from the normalized abnormal data and $\mathbf{C}$, which is obtained from known data. If newly monitored data is abnormal, the $\overline{M D}_{p}$ should be considerably greater than one.

$\overline{M D}_{p}={\overline{D^{2}}}_{p}=\frac{1}{k} \mathbf{Y}_{p}^{T} \mathbf{C}^{-1} \mathbf{Y}_{p}$

where $\mathbf{Y}_{p}=\left(y_{1}^{p}, y_{2}^{p}, \cdots, y_{k}^{p}\right)$.

The required conditions to utilize MTS are as follows: the number of variables $k$ of normal data is equivalent to that of abnormal data; the number of observation data $n$ is larger than that of variable $k$; and the standard deviation of normal data $\sigma_{i}$ is not zero.

This study utilizes the outliers crossing a threshold for structural damage diagnosis. In deciding the threshold, the largest and smallest values of MD taken from the cross-validation were removed, and the trimmed mean value was adopted as the threshold using (n-2) MD distances to reduce the effect of outliers on the MDs. The $n$-fold cross-validation was also adopted for assessing how the results of a statistic analysis will generalize to an independent data set (Bishop 2006).

\section{DAMAGE-SENSITIVE FEATURE}

The linear dynamic system can be modeled by the AR model (Ljung 1999, Kim et al. 2012) as

$y(k)+\sum_{i=1}^{p} a_{i} y(k-i)=e(k)$

where $y(k)$ denotes the output of the system, $a_{i}$ is the $i$-th order AR coefficient and $e(k)$ indicates the noise term.

The coefficient $a_{p}$ is related to the pole of the system because the $z$-transformation of Equation 5 can be written as

$Y(z)=H(z) E(z)=\frac{1}{1+\sum_{i=1}^{p} a_{i} z^{-i}} E(z)$

where $Y(z)$ and $E(z)$ are $z$-transformations of $y(k)$ and $e(k), H(z)$ is the transfer function of the system in the discrete-time complex domain, and $z^{-i}$ denotes the forward shift operator.

Values of $z$ in which the elements of the transfer function matrix show infinite values are the poles. This means that the denominator of the transfer function is the characteristic equation of the dynamic system, given as

$z^{p}+a_{1} z^{p-1}+a_{2} z^{p-2}+\cdots+a_{p-1} z+a_{p}=0$

The poles on the complex plane are associated with the frequency and damping constant of the dynamic system of structures, as follows:

$z_{k}=\exp \left(-h_{k} \omega_{k} \pm j \omega_{k} \sqrt{1-h_{k}^{2}}\right)$

where $h_{k}$ and $\omega_{k}$ are the damping constant and circular frequency, respectively, of the $k$-th mode of the system, and $j$ represents the imaginary unit.

The poles $z_{k}$ of Equation 6 are obtained by finding the roots of the AR coefficient polynomial in the denominator of $H(z)$. The number of poles in the $z-$ plane equals the AR model order. It should be noted that AR coefficients can be defined by sums and products of its roots $z_{k}$ in Equation 8 according to Vieta's formula (Bold 1982). In other words, AR coefficients are directly associated with the modal parameters such as $\omega_{k}$ and $h_{k}$ in Equation 8, and AR coefficients are also affected by damage. Therefore the parameter from AR coefficients is adopted as a damage-sensitive feature and defined as

$D I_{j}=\frac{\left|a_{1}\right|}{\sqrt{\sum_{i=2}^{j} a_{i}^{2}}}$

where $a_{i}$ denotes the $i$-th AR coefficient and $D I_{j}$ is the damage indicator (DI) that considers up to the $j$ th AR coefficient. Kim et al. (2013) show potential of damage detection of a scaled bridge utilizing the damage-sensitive feature through a laboratory moving-vehicle experiment.

\section{STRUCTURAL DAMAGE DIAGNOSIS BY OUTLIER DETECTION}

Damage detection of the bridge is carried out utilizing the DI and MTS. The $D I_{3}$ (Nair et al. 2006) is used in this study, since the first AR coefficient is the most dominant among the coefficients and the influence of higher order coefficients decreases drastically with respect to time (Kim et al. 2013).

The MTS requires a rather larger number of observations than the number of variables or sensors. However only a limited number of runs were carried out in the field experiment in order to reduce time and cost. Therefore this study utilized data from sensor groups to reduce the number of sensors which is considered as the number of variables in MTS since reducing the number of variables in MTS results in a 
similar effect of increasing the number of observations relatively.

\subsection{Continuous-span steel truss bridge}

In applying MTS to damage detection of the ninespan continuous bridge, Bridge-A, four different sensor groups are considered as shown in Figure 4: Group 1 utilizes all the observation data from the 7 observation points, 4 and 6 to 11, for the damage detection; Group 2 utilizes all the observation data from the 4 observation points, 4, 7, 9, 11; Group 3 utilizes the data from two adjacent observation points for detecting even the damage location; and Group 4 utilizes the observation points except the two observation points near the damaged member, points 3 and 5 in Group 3, to examine the feasibility of detecting damage location without considering data from the sensors near the damage. Examples of the measured acceleration responses before and after severing the member are shown in Figure 6.

Results of MTS considering Group 1 and Group 2 are shown in Figure 7, in which the red horizontal line denotes the threshold. Figure 7 shows that most of the MDs of damage cross the threshold, and indicates high possibility of anomalous events in the bridge. It is obvious that for Group 2 the probability of MDs of the $n$-fold cross-validation crossing the threshold is lower than that of Group 1, which indirectly proves the fact that more observations can provide more stable results. The same conclusion was deduced for the other sensor groups investigated and is omitted for brevity.

Detecting the damage location is carried out using Group 3, and MDs are plotted as shown in Figure 8a). Apparently, the probability of crossing the threshold was higher for the group containing observation points near the damaged member as shown in Figure $8 \mathrm{~b}$ ). The result of detecting the damage location is natural since the result considers the data of two additional sensors deployed near the damaged

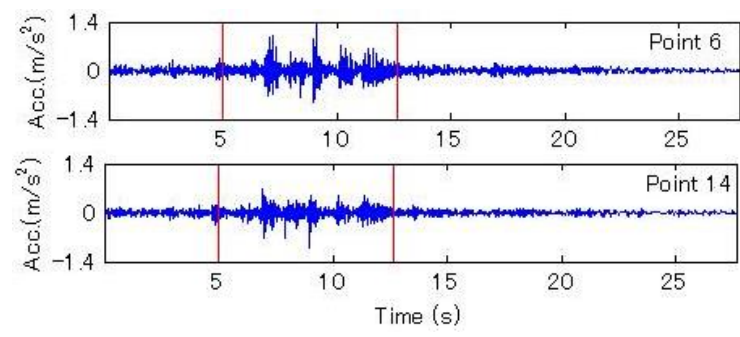

a)

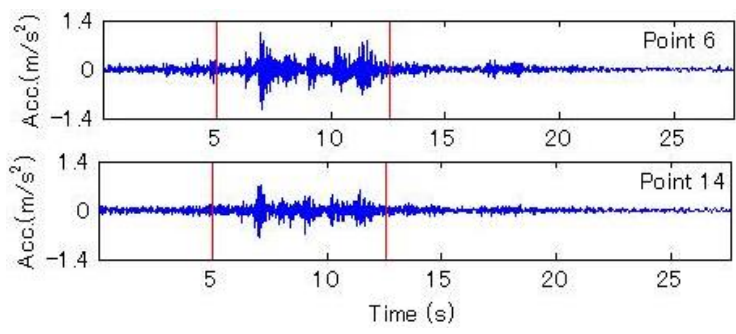

b)

Figure 6. Vehicle-induced vibrations of Bridge-A: a) Intact $(v=40 \mathrm{~km} / \mathrm{h}$, Run 1); b) Damage ( $v=40 \mathrm{~km} / \mathrm{h}$, Run 3 ). member, although it is a rather unrealistic idea to predict the damaged member and deploy sensors around that member for monitoring.

a)

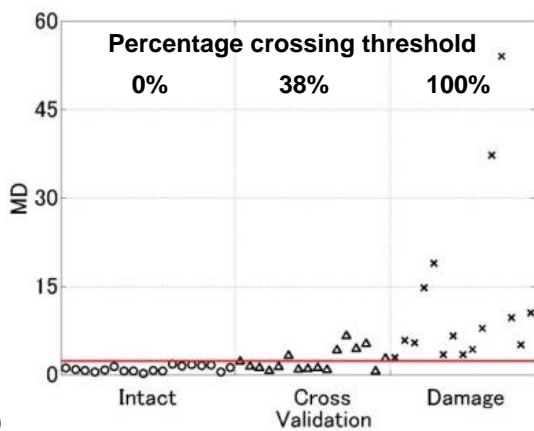

b)

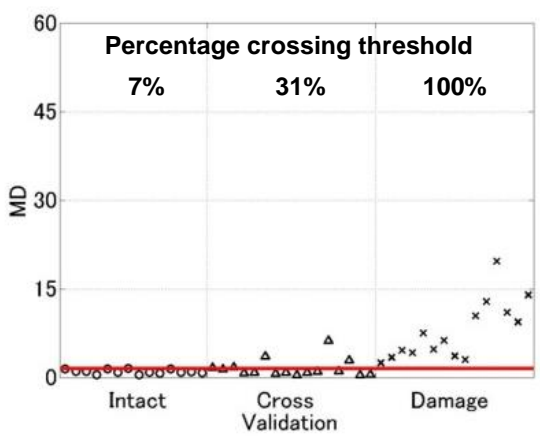

Figure 7. MDs of DI of Bridge -A: a) Group 1; b) Group 2.
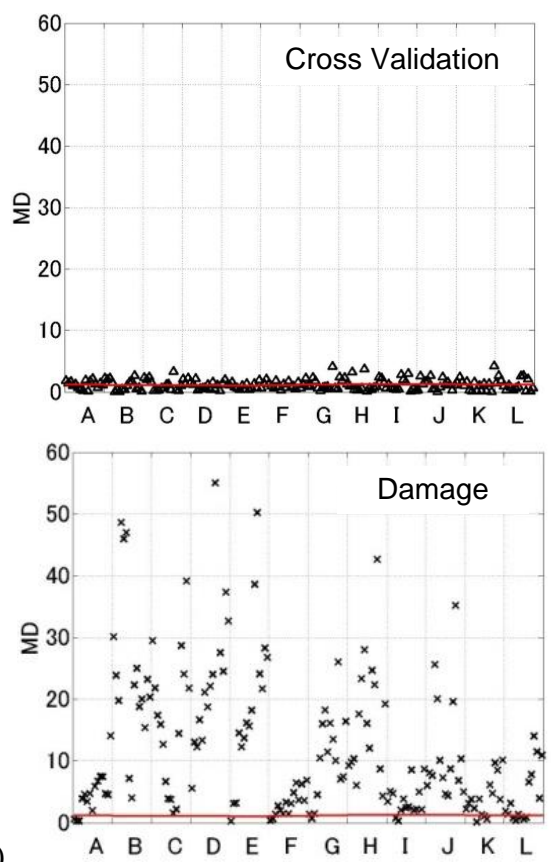

a)
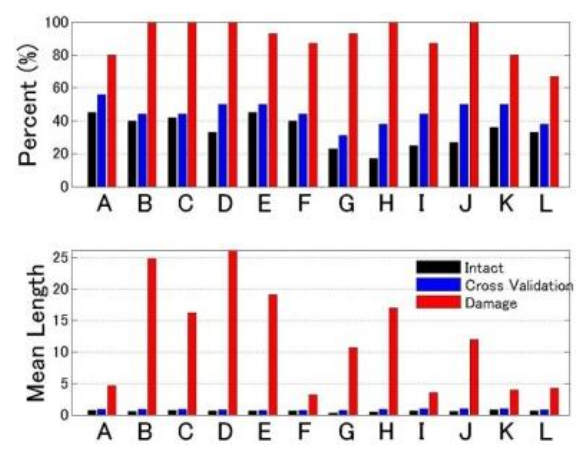

Figure 8. MTS results of Group 3 of Bridge-A: a) MDs; b) Percentage of MDs crossing the threshold, and mean length from the threshold of the MDs which cross the threshold. 

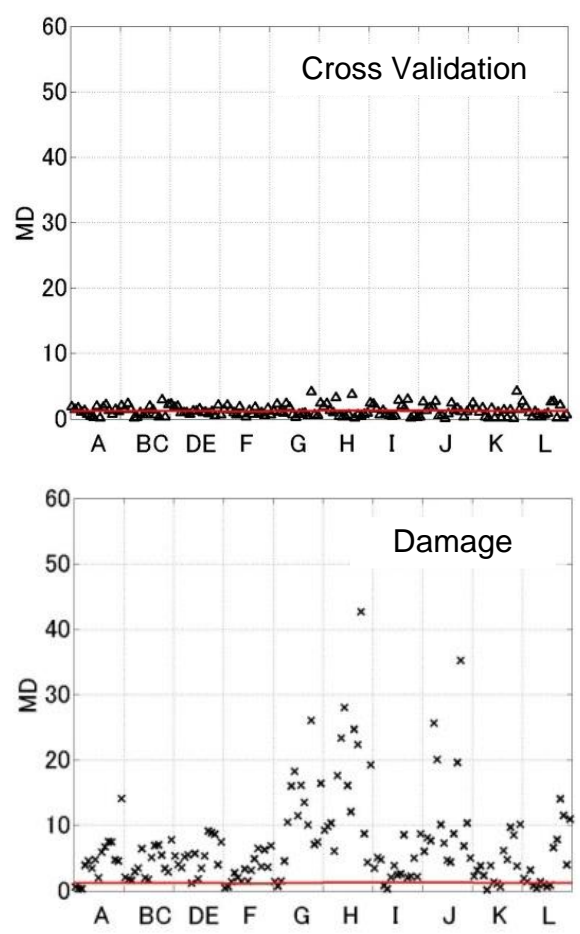

a)

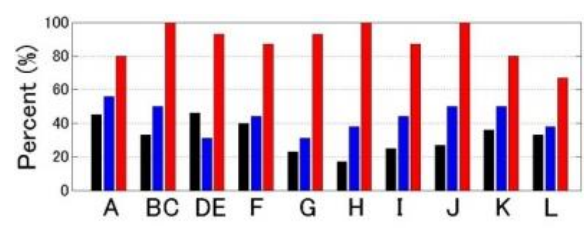

b)

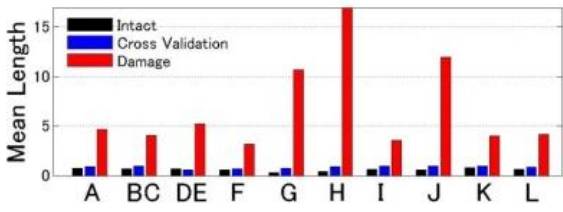

Figure 9. MTS results of Group 4 of Bridge-A: a) MDs; b) Percentage of MDs crossing the threshold, and mean length from the threshold of the MDs which cross the threshold.

This study also examines the feasibility of detecting damage location utilizing all observation points except the two observation points near the damaged member (Group 4). The results are summarized in Figure 9, which shows difficulty of identifying the damage location despite the results providing information about an anomaly in the observation data.

\subsection{Single-span steel truss bridge}

In applying MTS to damage detection of Bridge-B, 4 different sensor groups are considered as shown in Figure 5: Group 1 utilizes all the observation data from the 8 observation points for the damage detection; Group 2 utilizes five observation data from the observation points 1 to 5; Group 3 utilizes the data from the observation points 6 to 8; and Group 4 utilizes the data from two adjacent observation points for detecting damage location. Examples of the measured acceleration responses before and after severing the member are shown in Figure 10.

Results of MTS are shown in Figures 11 and 12. Observations from the Figures 11 and 12 show that most of the MDs crossed the threshold considering sensors of Group 2 and Group 3 for D-SCN1 and DSCN2, while for D-SCN3, Group 3 leads to high possibility to detect an anomaly. It proves the fact again that more observations can provide more stable results.

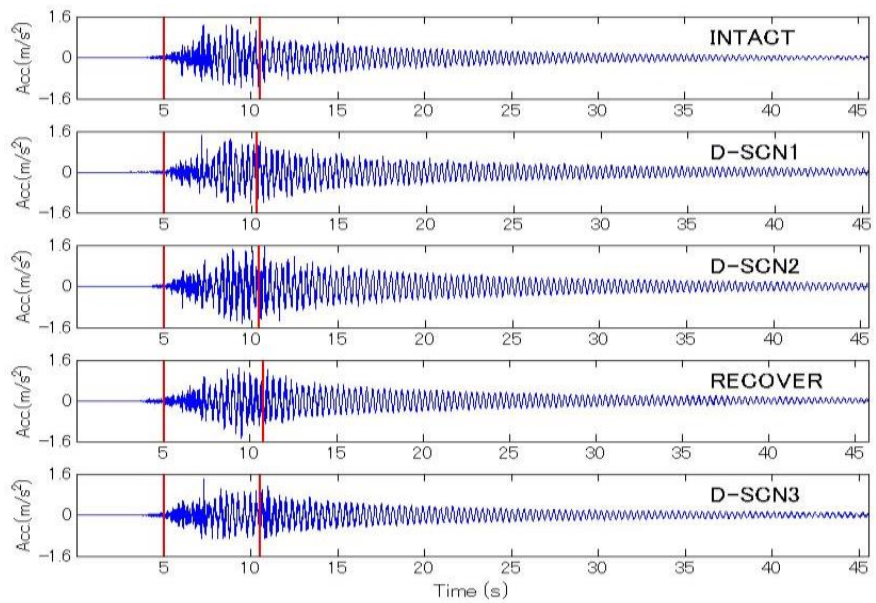

Figure 10. Vehicle-induced vibrations of Bridge-B under vehicle speed of $40 \mathrm{~km} / \mathrm{h}$.

a)

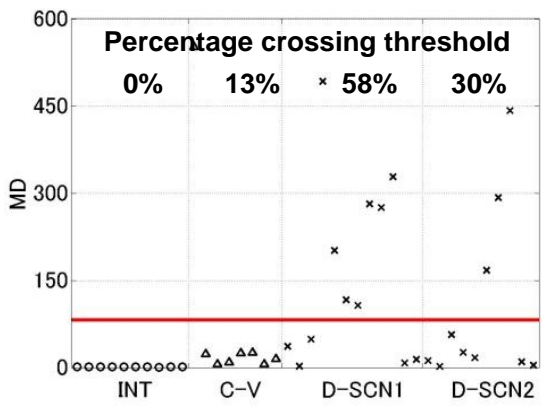

b)
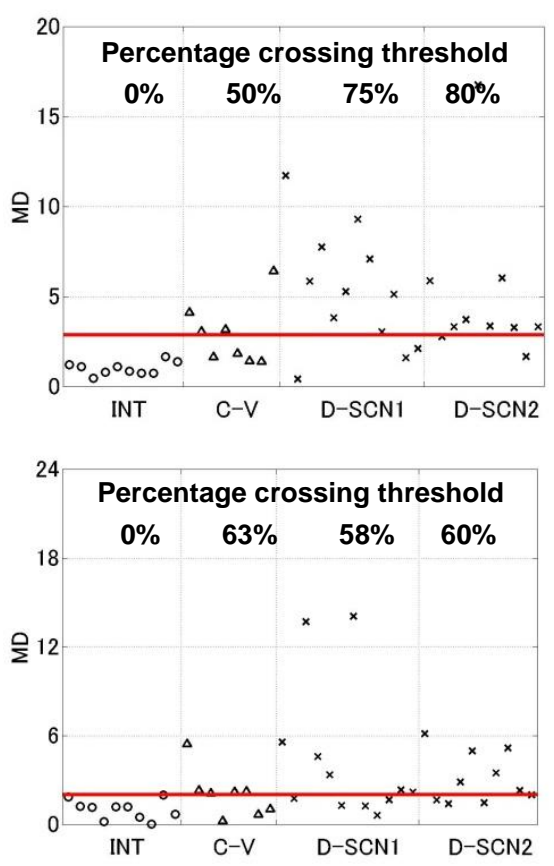

Figure 11. MDs of Bridge-B for D-SCN1 and D-SCN2: a) Group 1; b) Group 2; c) Group 3. 
a)

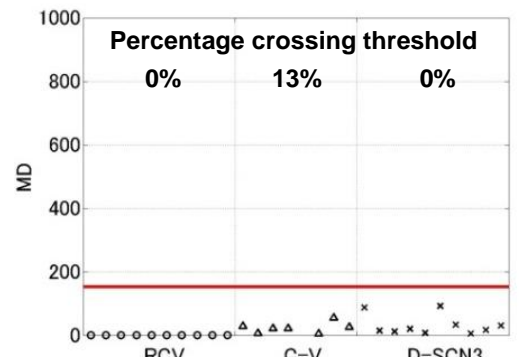

b)

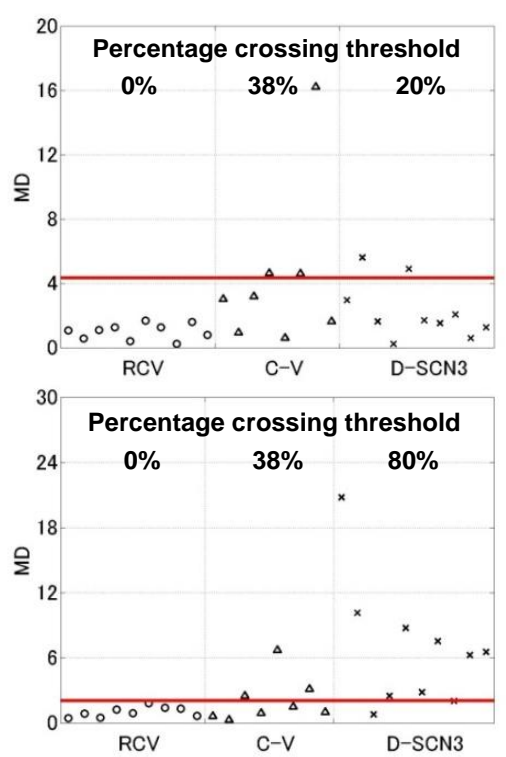

c)

Figure 12. MDs of Bridge-B for D-SCN3: a) Group 1; b) Group 2; c) Group 3.

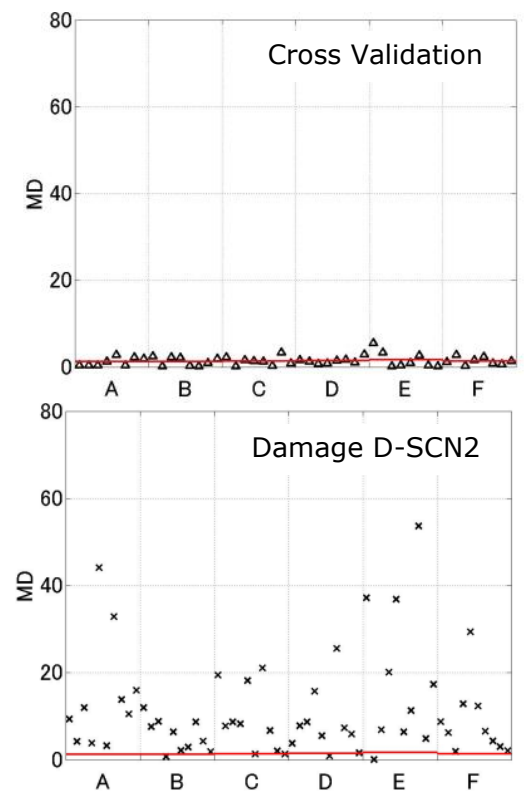

a)

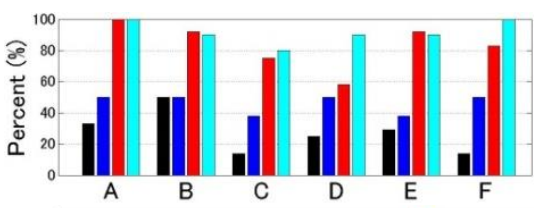

b)

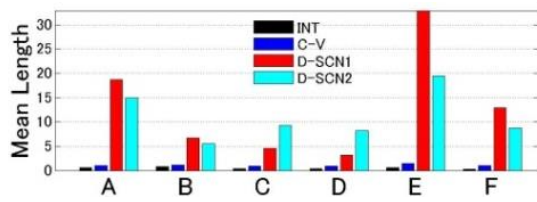

Figure 13. MTS results of Group 4 of Bridge-B for D-SCN1 and D-SCN2: a) MDs; b) Percentage of MDs crossing the threshold, and mean length from the threshold of the MDs which cross the threshold.

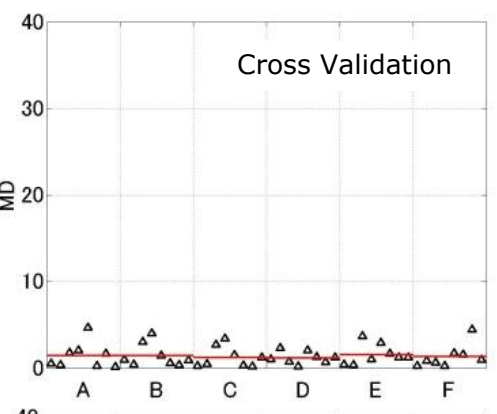

a)
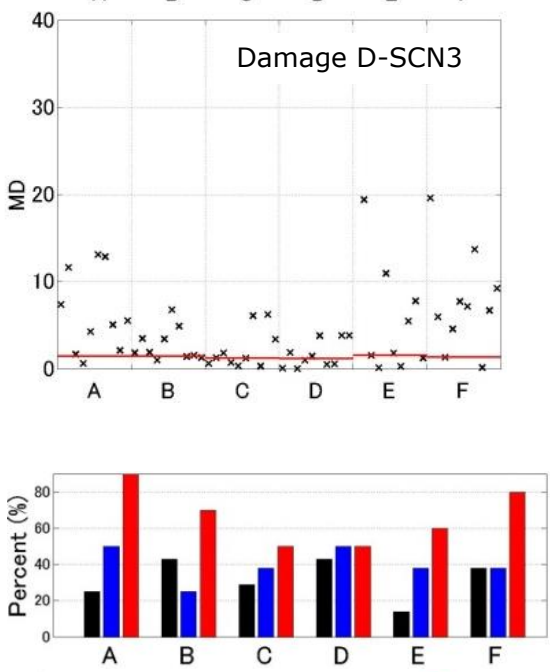

b)

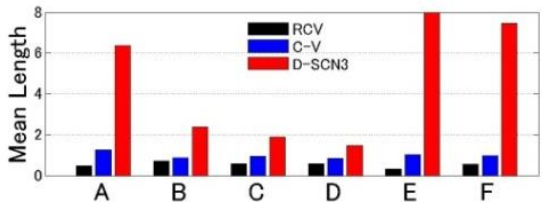

Figure 14. MTS results of Group 4 of Bridge-B for D-SCN3: a) MDs; b) Percentage of MDs crossing the threshold, and mean length from the threshold of the MDs which cross the threshold.

Detecting the damage location is carried out as summarized in Figures 13 and 14. However, it is hard to derive information relating to the damage location, despite the results providing information about an anomaly in the observation data.

\section{CONCLUDING REMARKS}

This study investigated the feasibility of vibrationbased bridge health monitoring of real steel truss bridges utilizing traffic-induced vibrations of the bridges. It considered linear system parameters of a time series model as a damage-sensitive feature. The Mahalanobis-Taguchi system (MTS) was used as a multivariate statistical fault detection to cope with any variation of identified results caused by uncertainties in the health monitoring and to emphasize potential change in the damage-sensitive feature due to damage. The Mahalanobis-distance (MD) which is obtained from probabilistic contours of data was used as a measure to detect an anomaly: i.e. probability of outliers crossing the threshold of MD as well as the mean length of MD from the threshold is considered. 
The damage indicator, a damage-sensitive feature derived from the coefficient of the AR model, combining with MTS was successfully applied to detect damage of the real steel truss bridges even utilizing the vehicle-induced vibration data. Denser sensor deployment near the damage member led to success in detecting damage location. However detection of damage location was unsuccessful after excluding the sensors near the damage member in the damage detection procedure. It also showed that more observations can provide more stable results from a statistical point of view.

Comparing results of Bridge-A (nine-span continuous bridge) with those of Bridge B (single-span bridge), mean values and probability of MD crossing the threshold of Bridge-A clearly showed higher possibility of detecting an anomalous event. One reason for the difference of accuracy in the statistical fault detection could be the severity of external loads since the external loading effect on vibrations of Bridge-A was more severe than those of Bridge-B.

The proposed damage detection approach which is based on the multivariate statistical fault detection is nonetheless applicable to BHM of real truss bridges. However, more comprehensive investigation is needed to clarify the reason for the failure to detect damage location of the single span bridge under a light moving vehicle and for the nine-span continuous bridge after removing the sensors near the damaged member under consideration. Future research will also focus on deciding the optimal number of sensors.

\section{ACKNOWLEDGEMENT}

This study is partly sponsored by JSPS, "Grant-inAid for Scientific Research (B) under project No. 24360178." The third author, K.C. Chang, is sponsored by "the JSPS Postdoctoral Fellowship for Foreign Researchers" Program. Such financial aids are gratefully acknowledged. The support from Osaka prefecture for the on-site experiment of the continuous-span steel truss bridge, and from Nara prefecture for the on-site experiment of the single-span steel truss bridge are also gratefully acknowledged.

\section{REFERENCES}

Bishop, C M. 2006. Pattern Recognition and Machine Learning, Springer.

Bold, B. 1982. Famous problems of geometry and how to solve them. New York, Dover.

Carden, E and Brownjohn, J. 2008. Arma modelled time-series classification for structural health monitoring of civil infrastructure. Mechanical Systems and Signal Processing, 22 (2): 295-314.

Catbas, FN and Aktan, AE. 2002. Modal analysis for damage identification: past experiences and Swiss Z-24 bridge, in: Proceedings of IMAC 20: International Modal Analysis Conference, Los Angeles, CA: 448-456.

Deraemaeker, A, Reynders, E, De Roeck, G and Kullaa, J. 2007. Vibration-based structural health monitoring using output-only measurements under changing environment, Mechanical Systems and Signal Processing, 22(1): 34-56.

Doebling, SW, Farrar, CR, Prime, MB and Shevitz, DW. 1998, A review of damage identification methods that examine changes in dynamic properties. Shock and Vibration Digest, 30(2): 91-105.

He, X and De Roeck, G. 1997. System identification of mechanical structures by a high-order multivariate autoregressive model. Computers and Structures, 64(1-4): 341-351.

Japan Road Association. 2009. A case studies on repairing and reinforcing highway bridges. Maruzen Co. Ltd. (in Japanese)

Kim, CW, Kawatani, M and Hao, J. 2012. Model parameter identification of short span bridges under a moving vehicle by means of multivariate AR model. Structure and Infrastructure Engineering, 8(5): 459-472.

Kim, CW, Isemoto, R, Sugiura, K, Kawatani, M. 2013. Structural fault detection of bridges based on linear system parameter and MTS method. J. of JSCE, JSCE, 1: 32-43.

Kopsaftopoulos, FP and Fassois, SD. 2010. Vibration based health monitoring for a lightweight truss structure: Experimental assessment of several statistical time series methods. Mechanical Systems and Signal Processing, 24(7): 19771997.

Lee, JJ and Yun, CB. 2006. Damage diagnosis of steel girder bridges using ambient vibration data. Engineering Structures, 28(6): 912-925.

Ljung, L. 1999. System identification-Theory for the user, 2nd Ed. PTR Prentice Hall, Upper Saddle River, M. J.

Nair, KK, Kiremidjian, AS and Law, KH. 2006. Time seriesbased damage detection and localization algorithm with application to the ASCE benchmark structure. J. of Sound and Vibration, 291(1-2): 349-368.

National Transportation Safety Board. 2008. Collapse of I-35W Highway bridge, Minneapolis, Minnesota, August 1, 2007. Highway Accident Report NTSB/HAR-08/03. Washington, DC.

Salawu, OS. 1997. Detection of structural damage through changes in frequency: A review. Engineering Structures, 19: 791-808.

Shinozuka, M, Yun, CB and Imai, H. 1982. Identification of linear structural dynamic systems. J. Engg. Mech. Div., ASCE, 108(6): 1371-1390.

Sohn, H, Farrar, C, Hunter, N and Worden, K. 2001. Structural health monitoring using statistical pattern recognition techniques. Journal of Dynamic Systems, Measurement, and Control, 123 (4): 706-711.

Taguchi, G and Jugulum, R. 2000. New trends in multivariate diagnosis. Indian Journal of Statistics, 62(B): 233-248.

Wang, L, Chan, THT, Thambiratnam, DP, Tan, ACC and Cowled, CJL. 2012. Correlation-based damage detection for complicated truss bridges using multi-layer genetic algorithms. Advances in Structural engineering, 15(5): 693706.

Yamada, K. 2008. An advice from rupture into a diagonal member of Kisogawa bridge. JSCE Magazine Civil Engineering, 93(1):29-30. (in Japanese)

Yoshioka, T, Takahashi, M, Yamaguchi, H and Matsumoto, Y. 2011. Damage Assessment of Truss Diagonal Members Based on Frequency Changes in Local Higher Modes. Procedia Engineering, 14: 3119-3126. 